\title{
Poster Sessions
}

P08.10.103

Acta Cryst. (2008). A64, C450

Polymorphism, isomorphism and variability in the inclusion chemistry of a diol host compound

Hong $\mathrm{Su}^{1}$, Luigi R Nassimbeni ${ }^{1}$, Edwin Weber ${ }^{2}$

${ }^{1}$ University of Cape Town, Chemistry, P.D.Hanh Building, Upper Campus, Chemistry Department, Uinversity of Cape Town, Cape Town, Western Cape Province, Rondebosch 7701, South Africa, ${ }^{2}$ Institut für Organische Chemie, Technische Universität, Bergakademie Freiberg, Leipziger Strasse 29, D-09596, Freiberg / Sachsen, Germany, E-mail : hong.su@uct. ac.za

The process of molecular recognition in inclusion compounds is governed by the intermolecular interactions which occur between the various host and guest molecules making up the resultant crystalline assembly. We have studied the diol host, 2,2'-bis (hydroxydiphenylmethyl-1,1'-binaphthyl(H), which crystallised in three different polymorphic structures and formed a series of inclusion compounds with pyridine $(\mathrm{P})$, morpholine $(\mathrm{M})$ and benzene(B). Different stoichiometries were obtained by manipulating the proportions of the guests and the crystallisation temperatures. Four of these inclusion compounds, H.3P, H.2P.M, H.2P.B and H.P.M.B, are isomorphous and the guests are located at fixed sites in the crystal structure. Their thermal analysis results were explained in terms of the host $\cdots$ guest interactions. The three polymorphs of the host compound show strong structural similarities as depicted by their Hirshfeld surface fingerprint plots, which exhibit dominant $\mathrm{H} \cdots \mathrm{H}$ interactions. The conformation of the host molecule remains essentially constant, and is governed by an intramolecular $\mathrm{O}-\mathrm{H} \cdots \mathrm{O}$ hydrogen bond in the polymorphs of the apohost and in all the nine inclusion compounds analysed.

Keywords: polymorphism, isomorphism, inclusion compound

\section{P08.10.104}

Acta Cryst. (2008). A64, C450

\section{New crystal forms of gabapentin}

Demetrius C. Levendis, Hayley Reece, Ahmed Shaikjee,

\section{Caryn Gamble}

University of the Witwatersrand, Chemistry, PO WITS 2050 , Johannesburg, Gauteng, 2050, South Africa, E-mail : demetrius.levendis@ wits.ac.za

The neuroleptic drug gabapentin exists in different crystal forms: metal salts, cocrystals or different polymorphs. Three monoclinic polymorphs which are stable at room temperature ${ }^{1}$ are described here. There is a considerable difference in the densities of the three polymorphs $\left(1.257,1.247\right.$ and $1.216 \mathrm{Mg} \mathrm{m}^{-3}$ for the $\alpha-, \beta$ - and $\gamma$ - forms respectively) which can be attributed to small differences in the molecular conformations, packing and intermolecular $\mathrm{N}-\mathrm{H}$ $\cdots \mathrm{O}=\mathrm{C}$ hydrogen bonds. Although centrosymmetric hydrogenbonded pairs can be identified in all of them, only the $\beta$-form exhibits intramolecular hydrogen bonding (Figure 1). Furthermore, small exo- or endo-therms have been observed by DSC at around $85^{\circ} \mathrm{C}$ when heating the $\beta$ or $\gamma$ forms, a phenomenon that appears to be associated with minute changes in the hydrogen bonded networks of these polymorphs.
Here, the similarities and differences in molecular conformation and packing of the gabapentin polymorphs, and new metal salts and cocrystals are compared.

[1] H.A. Reece and D.C. Levendis, Acta Cryst. (2008) C64, o105-o108.

Figure 1 . The centrosymmetric dimer of the $\beta$-polymorph of gabapentin.

Keywords: gabapentin, polymorph, cocrystal

\section{P08.10.105}

Acta Cryst. (2008). A64, C450

The effect of substituents on the occurrence of polymorphism in diaminodicyanopyrazine dyes

\section{Haruyuki Gontani, Shinya Matsumoto}

Yokohama National University, Department of Environment and Natural Sciences, Graduate School of Environment and Information Sciences., 79-2, Tokiwadai, Hodogaya-ku, Yokohama, Kanagawa, 240-8501, Japan, E-mail:d07ha027@ynu.ac.jp

2,5-Diamino-3,6-dicyanopyrazine dyes, characterized by intense fluorescence in solution and in the solid state, have been developed as functional dyes since 1990's. A series of derivatives with benzyl substituents on the amino groups have been found to have some conformational polymorphs with different colours. In parahalogenated derivatives (Figure), 1 was found to crystallize in three differently coloured polymorphs. On the other hand, 2 was found to crystallize in a yellow form in most cases. In this study, the effect of substituents on the occurrence of polymorphism was investigated by conformational searches and crystal structure predictions. The conformational searches showed that the terminal halogen substituents have no significant influence on the stability of the isolated conformers. Crystal structure predictions for the calculated conformers were then performed. The result revealed that the halogen substituents have strong influence on the relative crystal energies in the stable forms. The difference in a polymorph occurrence between $\mathbf{1}$ and 2 will be discussed on the basis of the calculated results.<smiles>[R20]Nc1nc(C#N)c([N+]([R])=O)nc1C#N</smiles>

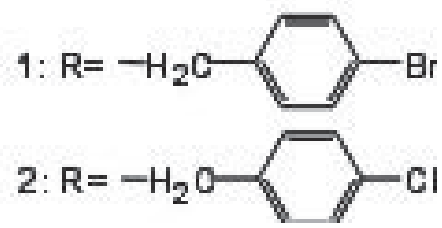

Keywords: polymorphism, crystal structure prediction, conformational analysis

\section{P08.10.106}

Acta Cryst. (2008). A64, C450-451

\section{Isomorphous pharmaceutical salts of lamotrigine with counterion dependence on water solubility}

Judit Galcera, Elies Molins

Institut de Ciencia de Materials de Barcelona (ICMAB-CSIC), Crystallography, Campus UAB, Bellaterra, Barcelona, 08193, Spain, E-mail:jgalcera@icmab.es

Lamotrigine is an anticonvulsant drug with low water solubility. In order to enhance it, lamotrigine salts of four different counterions were prepared. Six structures have been solved from single crystal $\mathrm{X}$-ray diffraction data. Four of these salts crystallized in $P 2_{1} / c$ 